\title{
Depression, Anxiety and Sexual Dysfunction in Patients with Diabetes Mellitus With and Without Foot Ulcer
}

\author{
Husin $\mathrm{H}$, Sidi $\mathrm{H}$, Baharudin A \\ Department of Psychiatry, Universiti Kebangsaan Malaysia Medical Centre (UKMMC), 56000 Cheras, Kuala \\ Lumpur, Malaysia.
}

\begin{abstract}
Introduction: Anxiety and depression are both associated with increased morbidity in diabetic population, especially those afflicted with foot ulcer, whereas Erectile Dysfunction ("ED") and Female Sexual Dysfunction ("FSD") are amongst the common complication found in diabetes. This study aimed to determine the prevalence of anxiety and depression as primary outcome and sexual dysfunction ("SD") as a secondary outcome in diabetic patients with and without foot ulcer ("DFU"). Methods: A cross-sectional study was conducted in Hospital Raja Permaisuri Bainun Ipoh, Perak, where 164 diabetic patients were recruited for the purpose of this study. Hospital Anxiety and Depression Scale ("HADS") were utilised to measure anxiety and depression. International Index for Erectile Function 5 ("IIEF-5") and the Malay version of Female Sexual Function Index (“MVFSFI") were used to detect SD. Simple and multiple logistic regressions were referred to in order to evaluate the factors associated with anxiety, depression and SD. Results: In the group with foot ulcer, $36.8 \%$ had depressive and anxiety risk respectively compared to without the foot ulcer, $37.5 \%$ and $38.5 \%$ respectively. Male gender $(A O R=6.3, p=0.002,95 \% \mathrm{Cl} 1.975,20.205)$ and having lower education status $(A O R=6.0, p=0.018,95 \% \mathrm{Cl} 1.36,26.59$, ) was associated with foot ulcer. High prevalence of $S D$ found in foot ulcer group $(52.6 \%, \mathrm{p}=0.006)$. Those with depression $(\mathrm{OR}=4.421,95 \% \mathrm{Cl} 1.08,18.16, \mathrm{p}=0.039)$ and male gender $(\mathrm{OR}=18.42, \mathrm{p}<0.01,95 \% \mathrm{Cl} 5.5,-61.62)$ predicts present of $S D$. Conclusion: Depression and anxiety prevalent were similar for both with and without diabetic foot ulcer. Several significant predictor factors were identified for DFU group, for anxiety and depression risk and for $S D$ in a diabetes patient. Further studies are required to establish the directional nature of this relationship.
\end{abstract}

Keywords: Depression, Anxiety, Diabetic food ulcer (DFU), Erectile Dysfunction (ED), Female Sexual Dysfunction (FSD)

\section{INTRODUCTION}

Diabetes Mellitus ("DM") is a metabolism disorder which leads to inappropriate hyperglycaemia either due to a reduction in the biologic effectiveness of insulin or an absolute deficiency of insulin secretion, or both. In Malaysia, the prevalence of diabetes has increased in the last decade from $11.6 \%$ in 2006 to $15.2 \%$ in 2011 to $17.5 \%$ (3.5 million) in 2015 among individuals 18 years and above. ${ }^{1}$ In general, Type 2 DM may result in various complications namely nephropathy, retinopathy, neuropathy and dermatopathy. ${ }^{2}$ About $60 \%$ of diabetic foot ulcers are the result of underlying neuropathy ${ }^{3}$ and vasculopathy, which retards the healing process. ${ }^{2}$ Diabetic foot ulcer ("DFU") is an open wound commonly located on the foot that occurs in about $15 \%$ of patient with diabetes and approximately $4.3 \%$ had lower limb amputation. ${ }^{2}$

Corresponding author:

Azlin Baharudin

Department of Psychiatry,

Universiti Kebangsaan Malaysia (UKMMC),

56000 Cheras, Kuala Lumpur, Malaysia.

Tel No: +60391455555 ext 6152

Fax No: +60391456681

Email Address:jelin72@hotmail.com

Tel No: +60391455555 ext 6152
Depression and anxiety may affect body metabolism in various ways. Studies showed stressful emotional experiences affect hormones release that may counter the insulin action (i.e. catecholamines, glucocorticoids, growth hormone and glucagons) and affect blood glucose level both in normal people and people with DM. Metaanalysis study showed depression was a risk factor for developing type 2 DM, where the increased risk was about $60 \%{ }^{4}$ However for type 1 diabetes, the prevalence is currently exhibiting insufficient evidence. ${ }^{5}$ For $D M$ patients with chronic foot ulceration, a study showed that this group had higher level of both depression and anxiety than in DM patients without complication. ${ }^{6}$

Generally for DM patient, previous studies showed prevalence of depression which varies from $11.5 \%,{ }^{7}$ $40.3 \%^{8}$ and up to $47.9 \%{ }^{6}$ While for DM patients with DFU, the prevalence rate of depression is much higher which ranges from $32.2 \%{ }^{9}$ up to $51.7 \% .{ }^{10}$ The prevalence of anxiety in DM population in a previous study showed that it varied from $30.5 \%,{ }^{7} 57.4 \%{ }^{11}$ up to $69.6 \%{ }^{6}$. While for anxiety in DM with DFU, a recent study showed its prevalence to be up to $36 \%{ }^{12}$

Sexual Dysfunction (SD) is a known diabetes-related complication that affects both male and female patients. SD can be potentially be associated in 
more people with DFU. For male with DM, it includes disorders of libido, ejaculatory problems, erectile dysfunction ("ED") and combination of the aforementioned conditions. The most common complaints reported was $E D$, about $35 \%-75 \%$ of patients with diabetes, compared to $26 \%$ in the general population. ${ }^{13}$ Meanwhile, prevalence of female sexual dysfunction ("FSD") was $18.2 \%{ }^{14}$ and the common SD conditions reported were lack of sexual desire, sexual dissatisfaction, orgasmic disorder, pain, arousal disorder and poor lubrication. ${ }^{13}$ There are few possible mechanisms resulting to these concerns; the first is through changes of blood supply to the urogenital tissue, which later will affect the genital lubrication. Secondly, DM also is known to cause neuropathic mediated alteration which affects the genital arousal response. ${ }^{13,14,15}$ Depressive symptoms were positively associated with $E D .^{16,17}$ For FSD, psychological factors that associated were fear, depression, fatigue, stress, anxiety and belief. ${ }^{18}$ Be that as it may, studies determining frequency of sexual dysfunction whether involving diabetic or nondiabetic women are largely limited. ${ }^{15}$ Previous local study done in women with DM population showed that the prevalence of FSD was up to $18.2 \%{ }^{14}$

In Malaysia, various studies have been carried out on the prevalence and risk factors of depression and anxiety among diabetics. However, the study on prevalence of depression and anxiety on diabetic patient with DFU is still lacking. In relation to sexual dysfunction, local data also limited especially for $E D$ and FSD for DM patients in the presence of foot ulcer. This study therefore aims to compare the prevalence of anxiety and depression in DM patient with and without DFU as its primary objective. The secondary outcome is to know the prevalence of sexual dysfunction in both groups and at the same time to identify their associated factors for all outcomes. This study may help us to understand more about factors associated with depression, anxiety and sexual dysfunction in DM patients with and without foot ulcer.

\section{MATERIALS AND METHODS}

\section{Ethical Consideration}

Ethics committee of the Faculty of Medicine, University Kebangsaan Malaysia (FF-2015-212) and National Medical Research and Ethics Committee (MREC) of the Ministry of Health Malaysia (MOH), via the National Medical Research Registry (NMRR) has approved the study prior to its commencement (NMRR-15-1043-25205). Referral to the specialist centre was offered to participants who were detected to have psychiatric morbidity and sexual dysfunction.

\section{Statistical Analysis}

The data was analysed using the International
Business Machine (IBM) Statistical Package for

Social Science (SPSS) version 23 (New York, USA). Numerical data was tested using Mann Whitney $U$ analysis. Categorical variables of interest and their associated factors were calculated using chi-square test. Relationship between outcome variables analysed using simple logistic regression analysis and multiple logistic regressions were run to determine the predictor of associated factor. Two tails with alpha (a) values of 0.05 were used in all tests.

\section{Study Design}

A cross sectional study was carried out in outpatient clinic of medical and orthopedic departments in Hospital Raja Permaisuri Bainun (HRPB) Ipoh, Perak, once to twice per week from January 2016 to May 2016. A simple systematic randomization was done whereby every patient who went to the registration counter for normal clinic follow-ups with even number, regardless of clinic room was approached. For an example, the numbers 3022, 3024, 3026, etc., where the number ' 3 ' indicated clinic room number while the rest are the number of respective patients. The inclusion criteria were: (i) with DM; (ii) ages between 18-60 years old; (iii) fluent in local Malay language (able to read and understand the spoken language) and (iv) had given consent for this study. The exclusion criteria were: (i) refusal to give consent and (ii) not fluent in local Malay language.

\section{Study Procedure}

Those who have fulfilled the inclusion criteria were given questionnaires on socio demographic and HADS questionnaire to detect any psychiatric morbidity. Individual who was sexually active, ie. having sexual intercourse for at least once a month, was asked to complete IIEF 5 (male participants who were sexually active for the past 6 months) and MVFSFI (female participants who were sexually active for the past 4 weeks). The questionnaires involved in this study were all self-reporting, and a researcher was arranged to assist any participant who needs further clarification about the questionnaire. Those who had recent and previous history of DFU at the time of the study will be allocated to the DFU group, based on the questionnaire and additional confirmatory records by the said hospital.

\section{Study Instrument}

1. A self-constructed questionnaire on demographic and brief clinical detailed;

The questionnaire was devised to obtain data such as gender, ethnicity, religion, marital status, education level, monthly household income, occupation and smoking status. The appropriate clinical data which were based on self-reporting included height $(\mathrm{m})$, weight $(\mathrm{kg})$, 
duration of $D M$ in years, other medical illness and DFU grading using Wagner's classification (Wagner WF, 1981). ${ }^{19}$ For DFU grading, it was done by the doctor in charge of those who had ulcer problem. Records of the ulcer severity were traced in the patients' files or were inquired directly to the treating doctor.

2. Hospital Anxiety Depression Scale ("HADS"); The Malay version of HADS is a valid screening tool for Malaysian population to identify depression and anxiety with sensitivity up to $90.0 \%$ and specificity of $86.2 \%$ for anxiety and sensitivity of $93.2 \%$ and specificity of $90.8 \%$ for depression with cutoff point $8 / 9 .{ }^{20}$ The reason this scale was referred to in this study was because it carries a lower risk of confounding diabetes-related and psychological morbidity symptoms compared to some other widely used scales. $^{21}$ The scale has been validated for the Malaysian population in previous study. ${ }^{22}$ It had good internal consistency reliabilities measured with Cronbach's alpha coefficient which were 0.86 and 0.78 for anxiety and depression respectively. The questionnaire had two sections - each with seven items for both Anxiety and Depression scale, which was rated using Likert scale; 0 (not at all) to 3 (almost always). The scores for anxiety and depression were categorized into three groups: (i) 0-7 "no risk", (ii) 8-10 "borderline risk" and (iii) $\geq 11$ as "intermediate or severe risk" for both. Other ways to describe the anxiety and depression scores are using the following cut points: a score between 0 and 7 was classified as normal and a score between 8 - 21 points as increased risk. This had been used in this study for analysis.

3. International Index for Erectile Function (IIEF 5); IIEF-5 is an abbreviated version of the IIEF 15 , which was the original instrument developed as a diagnostic tool for $E D$ to complement clinical judgment. The IIEF-5 is a subset of IIEF 15 and it comprises items 2, 4, 5, 7, and 15, specifically for erectile function ("EF"). The Malay version of the IIEF-5 was acceptable measurement as the area under the ROC curve of the Malay IIEF- 5 was 0.86 ; where the optimal cut-off score is 17 , and the sensitivity and specificity was $85 \%$ and $75 \%$ respectively. ${ }^{23}$ The items are rated on a 6 grade likert scale in which score $\leq 17$ indicates presence of $E D$.

4. Malay version of Female Sexual Function Index (MVFSFI);

MVFSFI is a 19-items questionnaire that is reliable and valid to be used among Malaysian community. ${ }^{24}$ Sexual dysfunction will be present in those who score a total of 55 and lower with 99\% sensitivity and $97 \%$ specificity. The following are the six domains of sexual function measured in MVFSFI and its cut-off score with sensitivity and specificity respectively: (i) desire; $\leq 5$ for sexual desire disorder (sensitivity = 95\%, specificity = 89\%); (ii) arousal; $\leq 9$ for sexual arousal disorder (sensitivity $=77 \%$, specificity $=$ 95\%); (iii) lubrication; $\leq 10$ for disorder of lubrication (sensitivity $=79 \%$, specificity $=87 \%$ ); (iv) orgasm; $\leq 4$ for orgasmic disorder (sensitivity $=83 \%$, specificity $=85 \%$ ); (v) satisfaction; $\leq 11$ for sexual dissatisfaction (sensitivity = 83\%, specificity $=85 \%$ ); and (vi) pain; $\leq 7$ for sexual pain disorder (sensitivity $=86 \%$, specificity $=$ $95 \%)^{24}$

\section{RESULTS}

A total of 960 patients were encountered and 480 patients have been approached via simple randomization (refer to figure 1). About $50 \%$ of them did not fulfil the inclusion criteria as they either have no DM; as this study was conducted in general orthopedic clinic which may involve other limb problems not related to DFU and also in general medical clinic; which comprised of patients with various diagnosis including infectious diseases (i.e. hepatitis, AIDS etc.), thyroid disease, hematological disorder, rheumatologic disorder and etc., or that they were in extreme age group; i.e. older than 65 years of age or younger than 18 years of age as well as some who did not comprehend Bahasa Melayu. $31.7 \%$ of those approached were uninterested and had therefore declined their participation in the study. A total of 164 participants have fulfilled the inclusion criteria and were recruited in the study. However only 118 samples were able to give complete details of their weight and height (either based on participant own knowledge or tracing from the case note) whereas $62.8 \%(n=103)$ of the participant answered the questionnaire on sexual dysfunction which comprised of 74 respondents who completed the IIEF 5 and 29 respondent who completed the MVFSFI. Those who had not completed the questionnaires were not sexually active.

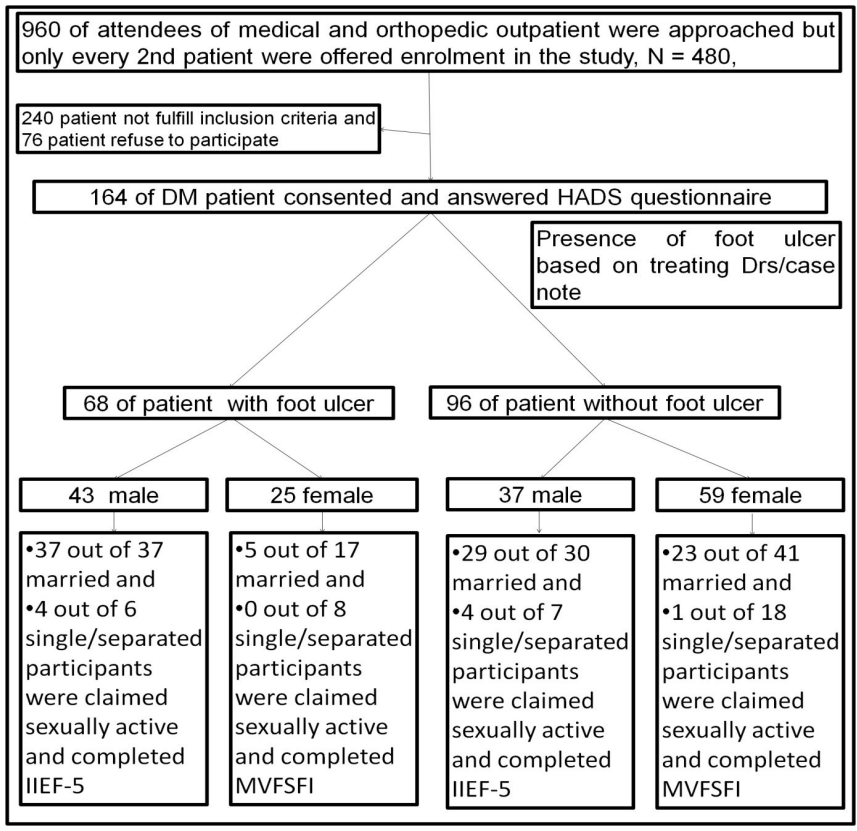

Figure 1 Flow chart of the study sample collections 


\section{Respondent characteristics}

The median age of the DM participants was 56.0 years old (IQR $=48-60.75)$ and comprised of $48.8 \%$ male and $51.2 \%$ female. Majority were Malay participants $(56.1 \%)$, followed by Indian (27.4\%), Chinese $(14.6 \%)$ and others $(1.8 \%)$. The same pattern is seen in the religious group, where Muslim (57.9\%) are followed by Hinduism (22.6\%), Buddhism (12.8\%), Christianity (4.3\%) and others (2.4\%). Majority of the participants were married $(76.2 \%)$ whereas $23.8 \%$ were either single or separated or divorced. $54.9 \%$ of participants' education level was limited up to secondary school and below, while 59.8\% were unemployed. $75 \%$ of the participants earned less than RM3000 and $90.2 \%$ of them were currently not smoking. The median duration of $D M$ for all samples was 10 years (IQR: $4-16$ ) and about $63.4 \%$ of samples had other medical co-morbidity. The medical co-morbidity includes hypertension (HPT) 55.5\%, Ischemic Heart Disease (IHD) $23.3 \%$ and others disease $29.9 \%$. Among all DM participants, $41.5 \%$ had DFU and in contrast, $58.5 \%$ had no DFU. $31 \%$ of participants, who gave their weight and height had obese BMI. Among those who had DFU, more than $50 \%$ had grade 3 and higher BMI (Table 1).

Table I: Sociodemographic characteristic \& prevalence risk of depression, anxiety \& SD in DM patient with and without DFU

\begin{tabular}{|c|c|c|c|c|c|c|c|c|}
\hline Presence DFU & Yes, N (\%) & No, $N(\%)$ & $P$ value & $\begin{array}{l}\text { Crude } \\
\text { OR }\end{array}$ & $95 \% \mathrm{Cl}$ & $\begin{array}{l}\text { Adjusted } \\
\text { OR }\end{array}$ & $95 \% \mathrm{Cl}$ & $P$ value \\
\hline $\begin{array}{l}\text { Total of } 164 \mathrm{DM} \\
\text { participant }\end{array}$ & $68(41.5)$ & $96(58.5)$ & & & & & & \\
\hline $\begin{array}{l}\text { Age } \\
\text { Med (IQR) }\end{array}$ & $56.0(51-63)$ & $55.5(47-60)$ & $\begin{array}{l}0.283^{*} \\
0.169\end{array}$ & 1.024 & $0.990-1.060$ & - & - & - \\
\hline Gender & & & 0.002 & 2.743 & $1.444-5.211$ & 6.317 & $1.975-20.205$ & 0.002 \\
\hline Male & $43(53.8)$ & $37(46.3)$ & & & & & & \\
\hline Female & $25(29.8)$ & $59(70.2)$ & & & & & & \\
\hline Ethnicity & & & 0.009 & 2.381 & $1.239-4.576$ & - & - & 1.00 \\
\hline $\begin{array}{l}\text { Malay } \\
\text { Non Malay }\end{array}$ & $\begin{array}{l}46(50) \\
21(29.6)\end{array}$ & $\begin{array}{l}46(50) \\
50(70.4)\end{array}$ & & & & & & \\
\hline Religion & & & 0.015 & 2.238 & $1.166-4.294$ & - & - & 1.00 \\
\hline $\begin{array}{l}\text { Muslim } \\
\text { Non Muslim }\end{array}$ & $\begin{array}{l}47(49.5) \\
21(30.4)\end{array}$ & $\begin{array}{l}48(50.5) \\
48(69.6)\end{array}$ & & & & & & \\
\hline Marital status & & & 0.420 & 1.358 & $0.646-2.858$ & - & - & - \\
\hline $\begin{array}{l}\text { married } \\
\text { Single/divorced }\end{array}$ & $\begin{array}{l}54(43.2) \\
14(35.9)\end{array}$ & $\begin{array}{l}71(56.8) \\
25(64.1)\end{array}$ & & & & & & \\
\hline Educational level & & & 0.077 & 2.028 & $0.926-4.441$ & 6.014 & $1.360-26.585$ & 0.018 \\
\hline $\begin{array}{l}\text { Secondary school and } \\
\text { lower }\end{array}$ & $57(45.2)$ & $69(54.8)$ & & & & & & \\
\hline $\begin{array}{l}\text { Higher than secondary } \\
\text { school }\end{array}$ & $11(28.9)$ & $27(71.1)$ & & & & & & \\
\hline Employment status & & & 0.041 & 1.975 & $1.029-3.791$ & 1.786 & $0.589-5.417$ & 0.306 \\
\hline $\begin{array}{l}\text { Employed } \\
\text { Unemployed }\end{array}$ & $\begin{array}{l}21(31.8) \\
47(48)\end{array}$ & $\begin{array}{l}45(68.2) \\
51(52)\end{array}$ & & & & & & \\
\hline Household income & & & 0.714 & 1.144 & $0.556-2.356$ & - & - & - \\
\hline $\begin{array}{l}\text { Less than RM3000 } \\
\text { More than RM3000 }\end{array}$ & $\begin{array}{l}52(42.3) \\
16(39)\end{array}$ & $\begin{array}{l}71(57.7) \\
25(61)\end{array}$ & & & & & & \\
\hline Smoking status & & & 0.212 & 1.939 & $0.685-5.493$ & - & - & - \\
\hline $\begin{array}{l}\text { Yes } \\
\text { No }\end{array}$ & $\begin{array}{l}9(56.3) \\
59(39.9)\end{array}$ & $\begin{array}{l}7(43.8) \\
89(60.1)\end{array}$ & & & & & & \\
\hline $\mathrm{BMI}$ in $\mathrm{kg} / \mathrm{m}^{2} \mathrm{n}=118$ & $\begin{array}{l}26.8(23.9- \\
29.9)\end{array}$ & $\begin{array}{l}28.2 \\
(24.5-34.0)\end{array}$ & 0.154 & - & - & - & - & - \\
\hline Obesity (BMI>30) & & & 0.094 & 1.988 & $0.890-4.490$ & - & - & - \\
\hline $\begin{array}{l}\text { Yes } \\
\text { No }\end{array}$ & $\begin{array}{l}13(35.1) \\
42(51.9)\end{array}$ & $\begin{array}{l}24(64.9) \\
39(48.1)\end{array}$ & & & & & & \\
\hline Medical Co morbid & & & 0.344 & 1.370 & $0.714-2.630$ & - & - & - \\
\hline $\begin{array}{l}\text { Yes } \\
\text { No }\end{array}$ & $\begin{array}{l}46(44.2) \\
22(36.7)\end{array}$ & $\begin{array}{l}58(55.8) \\
38(63.3)\end{array}$ & & & & & & \\
\hline $\begin{array}{l}\text { Duration of DM in } \\
\text { years }\end{array}$ & $\begin{array}{l}6.5(2.25- \\
10.0)\end{array}$ & $\begin{array}{l}10.0 \\
(5.0-15.0)\end{array}$ & $\begin{array}{l}0.787^{*} \\
0.833\end{array}$ & 1.004 & $0.966-1.044$ & - & - & - \\
\hline $\begin{array}{l}\text { Duration of HPT in } \\
\text { years }\end{array}$ & $\begin{array}{l}10.0(5.0- \\
16.8)\end{array}$ & $\begin{array}{l}10.0 \\
(4.0-15.0)\end{array}$ & $\begin{array}{l}0.019 " \\
0.036\end{array}$ & 0.936 & $0.879-0.996$ & 0.946 & $0.833-1.014$ & 0.116 \\
\hline $\begin{array}{l}\text { Duration of IHD in } \\
\text { years }\end{array}$ & $2.0(1.0-8.5)$ & $\begin{array}{l}5.0 \\
(3.0-10.0)\end{array}$ & $\begin{array}{l}0.041^{\prime \prime} \\
0.256\end{array}$ & 0.925 & $0.809-1.058$ & - & - & - \\
\hline $\begin{array}{l}\text { Others disease } \\
\text { duration in years }\end{array}$ & $5.0(1.5-13.0)$ & $\begin{array}{l}9.0 \\
(4.3-11.5)\end{array}$ & $\begin{array}{l}0.394 \\
0.844\end{array}$ & 0.993 & $0.926-1.065$ & - & - & - \\
\hline \multicolumn{9}{|l|}{ DFU grading } \\
\hline Grade 0 & $4(5.9)$ & & & & & & & \\
\hline Grade 1 & $11(16.2)$ & & & & & & & \\
\hline Grade 2 & $17(25.0)$ & & & & & & & \\
\hline Grade 3 & $15(22.1)$ & & & & & & & \\
\hline Grade 4 & $12(17.6)$ & & & & & & & \\
\hline Grade 5 & $9(13.2)$ & & & & & & & \\
\hline
\end{tabular}




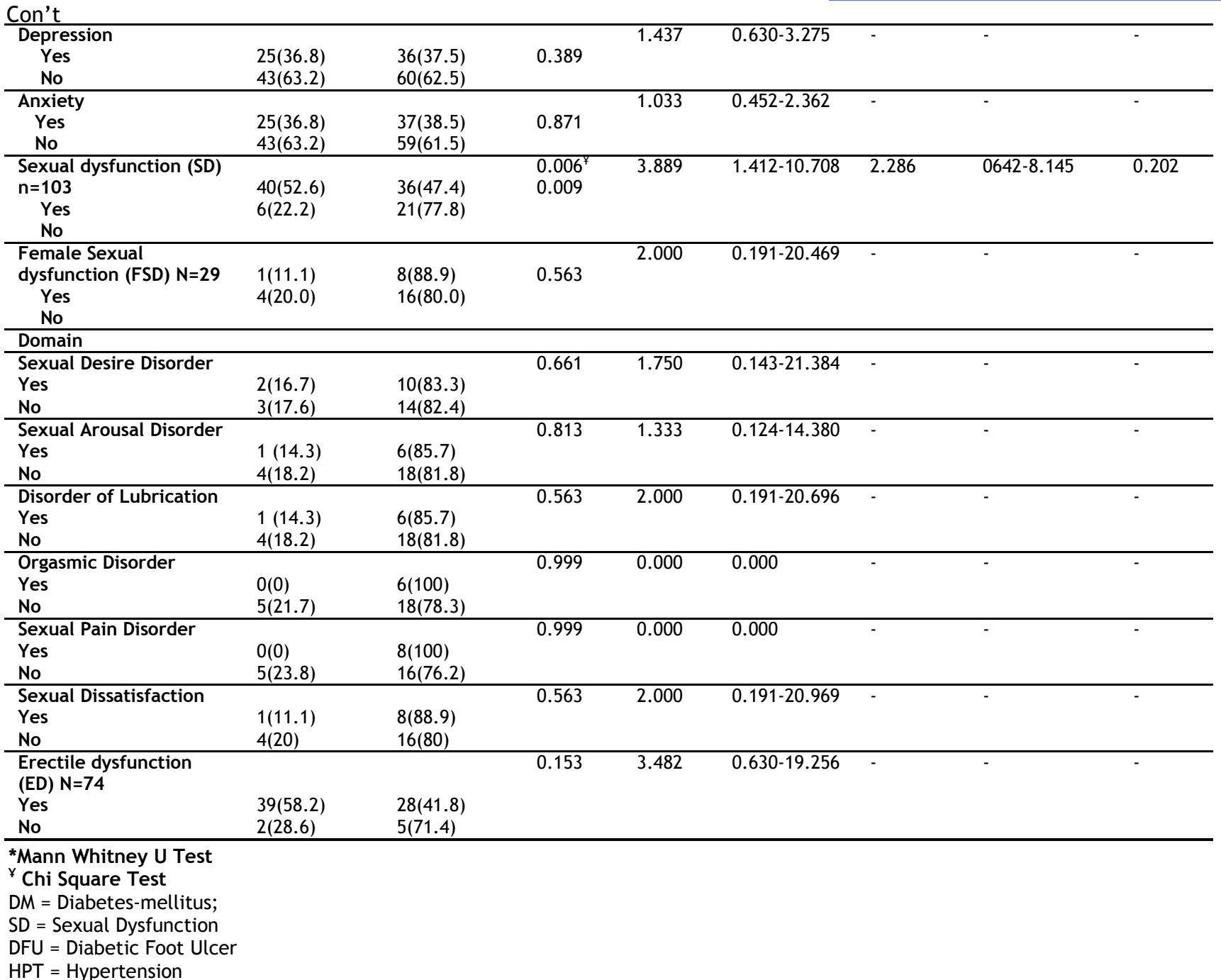

In the DFU group several patterns were noted which include more male than female participants $\mathbf{( 5 3 . 8 \%}$ versus $29.8 \%, p=0.002$, crude $O R=2.74,95 \% \mathrm{Cl} 1.44$, $5.21)$, more Malay than non-Malay participants $(50 \%$ versus $29.6 \%, \mathrm{p}=0.009$, crude $\mathrm{OR}=2.38,95 \% \mathrm{Cl} 1.24$, 4.58), similar pattern for Muslims than non-Muslims (49.5\% versus $30.4 \%, p=0.015$, crude $O R=2.24,95 \% \mathrm{Cl}$ $1.17,4.29)$ and more were unemployed than employed $(48 \%$ versus $31.8 \%, p=0.041$, crude $\mathrm{OR}=1.98,95 \% \mathrm{Cl} 1.03,3.79)$. Other differences in DFU group such as severity in having more than one medical co-morbidity, BMI less than $30 \mathrm{~kg} / \mathrm{m}^{2}$, smoking cigarette, married, having household income of less than RM3000 and education level of secondary school and lower, although these were found to be statistically insignificant.

There were significant differences for median duration of hypertension, HPT (10 years, IQR 5-12, $\mathrm{p}=0.02$ ) and duration of ischemic Heart Disease, IHD (4.5 years, IQR 1.75-10, $p=0.04$ ) between participants with DFU than without DFU. Among all factors, only male gender ( $p$ value $=0.013, O R=3.95$, $95 \% \mathrm{Cl} 1.33,11.70)$ and those with lower education status $(A O R=6.0, p$ value $=0.018,95 \% \mathrm{Cl} 1.36,26.59)$ were significantly associated with DFU.

Prevalence and risk factors for depression and anxiety among DM respondents
Table 1 also showed prevalence of depression (36.8\% and $37.5 \%$, $p$ value $=0.92)$ and anxiety $(36.8 \%$ and $38.5 \%, p$ value $=0.82$ ) of $D M$ patients with and without DFU respectively. Both results were statistically insignificant. Overall prevalence of depression and anxiety among DM participants regardless of the presence of foot ulcer were $37.2 \%$ and $37.7 \%$ respectively (Table 2 and 3 ). While among participants who had ED and FSD, 38.8\% and 44.4\% respectively had depression and anxiety symptoms. For those with depression, $18.9 \%$ has borderline risk and $18.3 \%$ has intermediate to severe risk. For DM participants who had anxiety, 25\% were borderline risk and $12.8 \%$ were intermediate to severe risk. Since the prevalence of depression and anxiety were insignificant between DM with and without DFU, thus the factors associated with depression and anxiety was carried out in all participants with DM.

Simple logistic regression analysis for DM patients with anxiety (refer table 3 ) showed significant determinant factors which comprises of (i) depression ( $p$ value $<0.01$, crude $O R=7.01,95 \% \mathrm{Cl}$ $3.46,14.23$ ), (ii) sexual pain disorder ( $p$ value $=0.03$, $\mathrm{OR}=7.08,95 \% \mathrm{Cl} 1.17,42.79$ ) (iii) unemployment ( $\mathrm{p}$ value $=0.01$, crude $\mathrm{OR}=2.66, \quad 95 \% \mathrm{Cl} 1.38$, 5.10) and (iv) cigarette-smoking participant ( $p$ value $=0.04, \mathrm{OR}=4.77,95 \% \mathrm{Cl} 1.05,21.77$ ) were found significantly associated with anxiety. 
Table II: Simple and multiple logistic analyses for factors associated with risk of depression in DM patient

\begin{tabular}{|c|c|c|c|c|c|}
\hline Characteristic & $\begin{array}{l}\text { Had Depression, } \\
\mathrm{N}(\%)\end{array}$ & $P$ value & $\begin{array}{l}\text { Crude OR }(95 \% \\
\mathrm{Cl})\end{array}$ & Adjusted OR(95\% Cl) & $P$ value \\
\hline All DM participant $(n=164)$ & $61(37.2)$ & & & & \\
\hline DFU & & 0.924 & $1.03(0.54-1.96)$ & - & - \\
\hline Yes & $25(36.8)$ & & & & \\
\hline No & $36(37.5)$ & & & & \\
\hline Anxiety & & $<0.001$ & $9.01(3.52-23.02)$ & $30.91(2.06-462.83)$ & 0.013 \\
\hline Yes & $40(64.5)$ & & & & \\
\hline No & $21(20.6)$ & & & & \\
\hline Sexual dysfunction (SD) $N=101$ & & 0.025 & $3.75(1.18-11.93)$ & - & 1.000 \\
\hline Yes & $30(39.5)$ & & & & \\
\hline No & $4(14.8)$ & & & & \\
\hline $\begin{array}{l}\text { Female Sexual dysfunction } \\
\text { (FSD) } N=29 \\
\text { Yes }\end{array}$ & $4(44.4)$ & 0.100 & $4.53(0.75-27.39)$ & - & - \\
\hline No & $3(15)$ & & & & \\
\hline Domain & & & - & - & - \\
\hline Sexual Desire Disorder & & 0.331 & & & \\
\hline Yes & $4(33.4)$ & & & & \\
\hline No & $3(17.6)$ & & & & \\
\hline Sexual Arousal Disorder & & 0.030 & $8.44(1.23-58.16)$ & - & 1.000 \\
\hline Yes & $4(57.1)$ & & & & \\
\hline No & $3(13.6)$ & & & & \\
\hline Disorder of Lubrication & & 0.442 & $2.00(0.34-11.70)$ & - & - \\
\hline Yes & $3(42.9)$ & & & & \\
\hline No & $4(18.2)$ & & & & \\
\hline Orgasmic Disorder & & 0.558 & $1.80(0.25-12.85)$ & - & - \\
\hline Yes & $2(33.3)$ & & & & \\
\hline No & $5(21.7)$ & & & & \\
\hline Sexual Pain Disorder & & 0.057 & $0.167(0.03-1.06)$ & - & - \\
\hline Yes & $4(50)$ & & & & \\
\hline No & $3(14.3)$ & & & & \\
\hline Sexual Dissatisfaction & & 0.016 & $11.25(1.58-80.30)$ & $7.680(0.11-555.68)$ & 0.351 \\
\hline Yes & $5(55.6)$ & & & & \\
\hline No & $2(10)$ & & & & \\
\hline Erectile dysfunction (ED) $\mathrm{N}=74$ & & 0.228 & $0.26(0.03-2.31)$ & - & - \\
\hline Yes & $26(38.8)$ & & & & \\
\hline No & $1(14.3)$ & & & & \\
\hline Gender & & 0.937 & $0.98(0.52-1.84)$ & - & - \\
\hline Male & $30(37.5)$ & & & & \\
\hline Female & $31(36.9)$ & & & & \\
\hline Ethnicity & & 0.137 & $0.61(0.32-1.17)$ & - & - \\
\hline Malay & $39(42.4)$ & & & & \\
\hline Non Malay & $22(31 \%)$ & & & & \\
\hline Religion & & 0.231 & $0.67(0.32-1.17)$ & - & - \\
\hline Muslim & $39(41.1)$ & & & & \\
\hline Non Muslim & $22(31.9)$ & & & & \\
\hline
\end{tabular}


Con't

\begin{tabular}{|c|c|c|c|c|c|}
\hline Marital status & & 0.187 & $0.61(0.30-1.27)$ & - & - \\
\hline Married & $43(34.4)$ & & & & \\
\hline Single/divorced & $18(46.2)$ & & & & \\
\hline Educational level & & 0.415 & $0.73(0.34-1.57)$ & - & - \\
\hline $\begin{array}{l}\text { Secondary school and } \\
\text { lower }\end{array}$ & $49(38.9)$ & & & & \\
\hline $\begin{array}{l}\text { Higher than secondary } \\
\text { school }\end{array}$ & $12(31.6)$ & & & & \\
\hline Employment status & & 0.420 & $0.77(0.40-1.46)$ & - & - \\
\hline Employed & $27(40.9)$ & & & & \\
\hline Unemployed & $34(34.7)$ & & & & \\
\hline Household income & & 0.227 & $1.60(0.75-3.43)$ & - & - \\
\hline Less than RM3000 & $49(29.3)$ & & & & \\
\hline More than RM3000 & $12((39.8)$ & & & & \\
\hline Smoking status & & 0.569 & $0.74(0.26-2.10)$ & - & - \\
\hline Yes & $7(43.7)$ & & & & \\
\hline No & $54(36.5)$ & & & & \\
\hline $\begin{array}{l}\text { Obesity }(\mathrm{BMI}>30) \\
\mathrm{N}=118 \\
\mathrm{Yes}\end{array}$ & $15(40.5)$ & 0.716 & $0.86(0.39-1.91)$ & - & - \\
\hline No & $30(37)$ & & & & \\
\hline Medical Co morbid & & 0.438 & $0.77(0.40-1.50)$ & - & - \\
\hline Yes & $41(39.4)$ & & & & \\
\hline No & $20(33.3)$ & & & & \\
\hline
\end{tabular}

Table III: Simple and multiple logistic analyses for factors associated with risk of anxiety in DM patient

\begin{tabular}{|c|c|c|c|c|c|}
\hline Characteristic & Had Anxiety & $P$ value & Crude OR $(95 \% \mathrm{Cl})$ & Adjusted $\mathrm{OR}(95 \% \mathrm{Cl})$ & $P$ value \\
\hline All DM participants & $62(37.7)$ & & & & \\
\hline DFU & & 0.817 & $1.08(0.57-2.05)$ & - & - \\
\hline Yes & $25(36.8)$ & & & & \\
\hline No & $37(38.5)$ & & & & \\
\hline Depression & & $<0.001$ & $7.01(3.46-14.23)$ & $8.67(3.96-18.97)$ & $<0.001$ \\
\hline Yes & $22(21.4)$ & & & & \\
\hline No & $40(65.6)$ & & & & \\
\hline Sexual dysfunction (SD) & & 0.112 & $0.44(0.16-1.21)$ & - & - \\
\hline Yes & $30(39.5)$ & & & & \\
\hline No & $6(22.2)$ & & & & \\
\hline $\begin{array}{l}\text { Female Sexual } \\
\text { dysfunction (FSD) } N=29\end{array}$ & & 0.301 & $0.42(0.08-2.20)$ & - & - \\
\hline Yes & $4(44.4)$ & & & & \\
\hline No & $5(25)$ & & & & \\
\hline Domain & & & - & - & - \\
\hline Sexual Desire Disorder & & 0.999 & & & \\
\hline Yes & $5(41.7)$ & & & & \\
\hline No & $4(23.5)$ & & & & \\
\hline $\begin{array}{l}\text { Sexual Arousal } \\
\text { Disorder }\end{array}$ & & 0.100 & $0.22(0.04-1.33)$ & - & - \\
\hline Yes & $4(57.1)$ & & & & \\
\hline No & $5(22.7)$ & & & & \\
\hline Disorder of Lubrication & & 0.858 & $0.86(0.16-4.62)$ & - & - \\
\hline Yes & $3(42.9)$ & & & & \\
\hline No & $6(27.3)$ & & & & \\
\hline Orgasmic Disorder & & 0.270 & $0.35(0.06-2.25)$ & - & - \\
\hline Yes & $3(50)$ & & & & \\
\hline No & $6(26.1)$ & & & & \\
\hline Sexual Pain Disorder & & 0.033 & $7.08(1.17-42.79)$ & $2.45(0.47-12.81)$ & 0.290 \\
\hline Yes & $5(62.5)$ & & & & \\
\hline No & $4(19)$ & & & & \\
\hline Sexual Dissatisfaction & & 0.065 & $0.20(0.04-1.11)$ & - & \\
\hline Yes & $5(55.6)$ & & & & \\
\hline No & $4(20)$ & & & & \\
\hline
\end{tabular}




\begin{tabular}{|c|c|c|c|c|c|}
\hline Erectile dysfunction & & 0.228 & $0.26(0.03-2.31)$ & - & - \\
\hline Yes & $26(38.8)$ & & & & \\
\hline No & $1(14.3)$ & & & & \\
\hline Gender $N=164$ & & 0.808 & $0.93(0.49-1.74)$ & - & - \\
\hline Male & $31(38.8)$ & & & & \\
\hline Female & $31(36.9)$ & & & & \\
\hline Ethnicity & & 0.514 & $0.81(0.43-1.53)$ & - & - \\
\hline Malay & $37(40.2)$ & & & & \\
\hline Non Malay & $25(35.2)$ & & & & \\
\hline Religion & & 0.723 & $0.89(0.46-1.53)$ & - & - \\
\hline Muslim & $37(38.9)$ & & & & \\
\hline Non muslim & $25(36.2)$ & & & & \\
\hline Marital status & & 0.394 & $0.73(0.35-1.51)$ & - & - \\
\hline Married & $45(36)$ & & & & \\
\hline Single/divorced & $17(43.6)$ & & & & \\
\hline Educational level & & 0.316 & $1.46(0.70-3.04)$ & - & - \\
\hline Secondary school and & $45(35.7)$ & & & & \\
\hline Higher than secondary & $17(44.7)$ & & & & \\
\hline Employment status & & 0.003 & $2.66(1.38-5.10)$ & $3.20(1.49-6.91)$ & 0.03 \\
\hline Employed & $34(51.5)$ & & & & \\
\hline Unemployed & $28(28.6)$ & & & & \\
\hline Household income & & 0.195 & $0.62(0.30-1.28)$ & - & - \\
\hline Less than RM3000 & $43(35)$ & & & & \\
\hline More than RM3000 & $19(46.3)$ & & & & \\
\hline Smoking status & & 0.044 & $4.77(1.05-21.77)$ & $8.51(1.59-45.56)$ & 0.01 \\
\hline Yes & $2(12.5)$ & & & & \\
\hline No & $60(40.5)$ & & & & \\
\hline Obesity $(\mathrm{BMI}>30) \mathrm{N}=118$ & & 0.532 & $1.16(0.60-2.22)$ & - & - \\
\hline Yes & $15(40.5)$ & & & & \\
\hline No & $28(34.6)$ & & & & \\
\hline Medical Co morbid & & 0.660 & $1.16(0.60-2.22)$ & - & - \\
\hline Yes & $38(36.5)$ & & & & \\
\hline No & $24(40)$ & & & & \\
\hline
\end{tabular}

However, multiple logistic regression analysis showed that (i) depressive symptom ( $p$ value $<0.01$, adjusted $\mathrm{OR}=8.67, \quad 95 \% \mathrm{Cl}$ 3.96-18.97), (ii) unemployment $(\mathrm{p}$ value $=0.03$, adjusted $O R=3.20$, $95 \% \mathrm{Cl} 1.49,6.91)$ and (iii) smoking cigarette ( $\mathrm{p}$ value $=0.01$, adjusted $\mathrm{OR}=8.51,95 \% \mathrm{Cl} 1.59,45.56$ ) were significant determinant for anxiety in $D M$ participant.

Meanwhile, anxiety ( $\mathrm{p}$ value $<0.01$, crude $O R=9.01$, $95 \% \mathrm{Cl} 3.52,23.02)$, sexual dysfunction (p value $=0.03$, crude $\mathrm{OR}=3.75,95 \% \mathrm{Cl} 1.18,11.93$ ), sexual arousal disorder ( $\mathrm{p}$ value $=0.03$, crude $\mathrm{OR}=8.44, \quad 95 \% \quad \mathrm{Cl} 1.23, \quad 58.16)$ and sexual dissatisfaction disorder ( $p$ value $=0.02$, crude $\mathrm{OR}=11.25,95 \% \mathrm{Cl} 1.58-80.30$ ) were significantly associated in $D M$ respondent with depression. From these factors, when further analysis was carried out via multiple logistic regression only anxiety ( $p$ value $=0.01$, adjusted $\mathrm{OR}=30.91,95 \% \mathrm{Cl} 2.06,462.83$ ) was found to be a significant determinant factor for depression (refer table 2).

Prevalence \& Risk of sexual dysfunction among respondent

Sexual dysfunction was more prevalent in DFU group $(52.6 \%$ versus $47.4 \%, p$ value $=0.01)$ whereas there is more ED in DFU group compared to non DFU group
(58.2\% versus $41.8 \%, p$ value $=0.13$ ) (refer to table 1). In male participants, 41 out of $43(95.3 \%)$ in DFU group and 33 out of $37(89.2 \%)$ in non DFU group responded to IIEF 5 questionnaire. Despite the finding not being statistically significant, it could be clinically significant as $E D$ is a consequence of poor glycemic control and peripheral neuropathy in $D M$ patient, ${ }^{25}$ its prevalence will be more in DFU group as DFU is also a consequence of peripheral neuropathy ${ }^{3}$, vasculopathy and poor glycemic control. ${ }^{26}$

In contrast,FSD was found to be more prevalent in non DFU group compared to DFU group $(88.9 \%$ versus $11.1 \%, p$ value $=0.56$ ). Both findings were not statistically significant. The reason for this insignificant finding could be due to small sample size for FSD $(n=29)$ as primarily the sample size calculated was based on prevalence of depression in $D M$ with or without DFU, and had not considered the prevalence of sexual dysfunction in $D M$ with or without DFU. Concluding FSD to be more prevalent in non DFU group is quite misleading as there are more who answered MVFSFI questionnaire in non DFU group, 24 out of $59(40.7 \%)$ than in DFU group, 5 out of $25(20.0 \%)$. The reason was many of them in DFU group are not sexually active especially female; hence they didn't answer the questionnaire. Therefore, the finding is only 
significant when both male and female data were combined for $S D$ and the results are only applicable for this study population rather than the general Malaysian population. In future, specific study can be done on sexual dysfunction with larger sample size.

For DM population, prevalence of SD was $73.8 \%$, in which specifically for ED and FSD were $31 \%$ and $90.5 \%$ respectively (refer table 4). Among participants who had ED and FSD, 38.8\% and $44.4 \%$ respectively had depression and anxiety symptoms. Presence of DFU (p value $=0.01$, crude $O R=3.89,95 \%$ $\mathrm{Cl} 1.41,10.71$ ), depressive symptoms ( $p$ value $=0.02$, crude $\mathrm{OR}=3.75,95 \% \mathrm{Cl} 1.18,11.93$ ) and male gender ( $\mathrm{p}$ value $<0.01$, crude $\mathrm{OR}=21.27,95 \% \mathrm{Cl} 7.03,64.33$ ) were factors significantly associated with SD. From these factors, only depressive symptoms ( $p$ value $=0.025$, adjusted $\mathrm{OR}=4.42,95 \% \mathrm{Cl} 1.08,18.16$ ) and male gender ( $\mathrm{p}$ value $=0.001$, adjusted $\mathrm{OR}=18.42,95 \% \mathrm{Cl} 5.51,61.62$ ) were more likely to have $S D$.

Table IV: Simple and multiple logistic analyses for factors associated with SD in DM patient

\begin{tabular}{|c|c|c|c|c|c|c|c|c|}
\hline Factors & Had SD, N (\%) & $P$ value & Crude OR & $95 \% \mathrm{Cl}$ & $P$ value & AOR & $95 \% \mathrm{Cl}$ & $P$ value \\
\hline DM participants $\mathrm{N}=103$ & $76(73.8)$ & & & & & & & \\
\hline DFU presence & & 0.006 & 3.89 & $1.41-10.71$ & 0.009 & 1.87 & $0.53-6.68$ & 0.333 \\
\hline Yes & $40(87.0)$ & & & & & & & \\
\hline No & $36(63.2)$ & & & & & & & \\
\hline Depression & & 0.019 & 3.75 & $1.18-11.93$ & 0.025 & 4.42 & $1.08-18.16$ & 0.039 \\
\hline Yes & $30(88.2)$ & & & & & & & \\
\hline No & $46(66.7)$ & & & & & & & \\
\hline Anxiety & & 0.106 & 2.283 & $0.83-6.31$ & 0.112 & - & & - \\
\hline Yes & $30(83.3)$ & & & & & & & \\
\hline No & $46(68.7)$ & & & & & & & \\
\hline Gender & & $<0.001$ & 21.27 & $7.03-64.33$ & $<0.001$ & 18.42 & $5.51-61.62$ & $<0.001$ \\
\hline Male & $67(90.5)$ & & & & & & & \\
\hline Female & $9(31)$ & & & & & & & \\
\hline Ethnicity & & 0.809 & 0.895 & $0.37-2.19$ & 0.809 & - & - & - \\
\hline Malay & $14(24.6)$ & & & & & & & \\
\hline Non Malay & $12(26.7)$ & & & & & & & \\
\hline Religion & & 0.432 & 0.702 & $0.29-1.70$ & 0.433 & - & - & - \\
\hline Muslim & $14(23.3)$ & & & & & & & \\
\hline Non Muslim & $13(30.2)$ & & & & & & & \\
\hline Marital status & & 0.776 & 0.789 & $0.15-4.05$ & 0.766 & - & - & - \\
\hline married & $25(26.6)$ & & & & & & & \\
\hline Single/divorced & $2(22.2)$ & & & & & & & \\
\hline Educational level & & 0.541 & 0.737 & $0.28-1.97$ & 0.542 & - & - & - \\
\hline $\begin{array}{l}\text { Secondary school and } \\
\text { lower }\end{array}$ & $19(24.7)$ & & & & & & & \\
\hline $\begin{array}{l}\text { Higher than secondary } \\
\text { school }\end{array}$ & $8(30.8)$ & & & & & & & \\
\hline Employment status & & 0.885 & 0.937 & $0.39-2.27$ & 0.885 & - & - & - \\
\hline Employed & $12(25.5)$ & & & & & & & \\
\hline Unemployed & $15(26.8)$ & & & & & & & \\
\hline Household income & & 0.582 & 0.758 & $0.28-2.04$ & 0.583 & - & - & - \\
\hline Less than RM3000 & $20(27.8)$ & & & & & & & \\
\hline More than RM3000 & $7(22.6)$ & & & & & & & \\
\hline Smoking status & & 0.275 & 0.427 & $0.09-2.04$ & 0.287 & - & - & - \\
\hline Yes & $2(14.3)$ & & & & & & & \\
\hline No & $25(28.1)$ & & & & & & & \\
\hline Obesity $(\mathrm{BMI}>30)$ & & 0.213 & 1.93 & - & - & - & - & - \\
\hline Yes & $14(60.9)$ & & & & & & & \\
\hline No & $42(75)$ & & & & & & & \\
\hline Medical Co morbid & & 0.696 & 1.20 & - & - & - & - & - \\
\hline Yes & $51(75)$ & & & & & & & \\
\hline No & $25(71.4)$ & & & & & & & \\
\hline
\end{tabular}




\section{DISCUSSION}

Diabetis is a chronic debilitating illness which can cause altered peripheral sensation of the foot, thus leading to actual ulceration process which may happen much earlier. Diabetic neuropathy and vasculopathy occurred when blood sugar is poorly controlled for prolonged periods of time. ${ }^{3}$ Majority of those with DFU had DM for more than 10 years. ${ }^{27}$ It is important to detect depression and anxiety in DM population as a whole as it affect compliance with the treatment regime leading to lower selfefficacy and to self-neglect, associated with higher morbidity, mortality and lower quality of life, even more in DFU group. ${ }^{28,29}$

In this study it was shown that significant characteristic of DFU group were more male gender, Malay ethnicity, Muslim religion, unemployed and have lower educational status. Out of these features, only male and lower education status was more predictive for DFU. Findings was similar to one study done in Portugal, whereby in DFU group, males were two times more likely than female to be scheduled for amputation and more than half were retired and majority had lower education status. ${ }^{30}$ However other study done by Mazlina et al locally and another study done in Egypt found no significant difference in terms of gender between patients with and without DFU. ${ }^{27,} 29$ Other possible reason could be because there are more male participants, 43 out of $68(63.2 \%)$ compared to female participants, 25 out of $68(36.8 \%)$ in DFU group of this study.

In large epidemiological study done in Norway involving more than 60,000 participants with 3 arm designs, those without $D M, D M$ without foot ulcer and $D M$ with history of foot ulcer, found that levels of anxiety and depression and psychological wellbeing did not differ between the two diabetes groups. ${ }^{31}$ Hence, the risk of depression and anxiety were similar regardless of the presence of DFU. Nevertheless, for DM population, the prevalence rate in this study was comparable to previous study done locally, which was $40.3 \%$ for depression, and $31.4 \%$ for anxiety. ${ }^{8}$ Specifically for DFU group, prevalence of depression in this study was still comparable to a study done in London $(32.2 \%)^{9}$ and in Australia (51.7\%). ${ }^{10}$ While for anxiety in DFU group, prevalence in current study was also comparable to study done in Italy (36\%). ${ }^{12}$ Regarding to the unemployment rate, this was understandable as more DFU patients were not able to continue with their current work because of the impact of DFU itself. Futhermore, the median age of DFU group was 56 years old, and most of the samples were already retired and not working anymore. Study done by Mazlina M et al in 2011 also found that there were more unemployed participants in DFU although the difference was not statistically significant. ${ }^{29}$

Current study showed that there were no statistical difference between prevalence of depression and anxiety for DM patient with and without DFU. The primary interest of this study was to look for depression and anxiety risk in diabetes with foot ulcer and found it was similar to diabetes group without foot ulcer. The findings could give the impression that the presence of foot ulcer has nothing to do with depression and anxiety for DFU group and should not be generalized for Malaysian population. This is because the study was conducted only in one centre; therefore a multicentre study in future could be the better way to do the research.

For DM with risk of depression, anxiety was a significant determinant factor. This finding was similar with previous study done locally by Kaur et al 2013. Although the result was statistically significant, the broad $\mathrm{Cl}$ gave negative impact on the result, i.e. the findings were less accurate. Therefore, the result needs to be interpreted carefully. On the other hand, those who had depressive symptom and unemployment were likely to have risk of anxiety in DM participant. Kaur et al 2013 found that unemployed DM patients were 1.2 to 1.6 times more likely to experience anxiety. Those who smoke cigarettes have more than 8 times higher risk to have anxiety in DM population, which is similar to previous study done in Ireland which found that smoking was a risk factor for higher anxiety in DM population. ${ }^{28}$

While for SD, its prevalence was found to be statistically significant in DFU group. DFU, FSD and ED were known complications of DM. This can be explained by the fact that DM may affect the neurovascular system (neuropathy and vasculopathy) of the respective organs. For FSD, the current study's finding was higher (31\%) as compared to previous local study done in women with DM population $18.2 \% .^{14}$ This is possible as prevalence of DM in Malaysia has been increasing year by year $^{1}$ and the current study combined groups of DM with and without DFU, while the previous study was done only in DM patient without DFU. Therefore, when looking at the prevalence of FSD in DM without DFU; it was $88.9 \%$ while for DM with DFU, its only $11.1 \%$ and the results were not significant.

Again the small sample size $(\mathrm{n}=29)$ who responded to the MVFSFI questionnaire may lead for thebiased result. However, the result is still comparable to previous study done in Italy which showed prevalence of FSD in DM women without complication as high as $75 \%{ }^{32}$ Researchers also identified that the flaw of MVFSFI questionnaire was due to its design which was meant only for those who are sexually active; those who are not sexually active were not able to answer the questionnaire. Therefore it is difficult for the researcher to comment on this factor, as most who are not answering the MVFSFI questionnaire in DFU group are probably having the FSD more than non DFU group that could cause them to become nonsexually active. 
On the other hand, for ED cases; previous study showed its prevalence in DM population range from $27 \%$ to $90 \% .13,33,34,35$. This study found higher prevalence of $90.5 \%$ of ED combining both DM patients with and without DFU, which is still comparable to previous findings. When looking at the separate groupings, prevalence of ED in DFU was only $58.2 \%$, which is higher compared to $41.8 \%$ in group without DFU. This was expected as DFU is a complication of peripheral neuropathy, as previous study showed those with peripheral neuropathy, the odds of having ED was $5.22(95 \% \mathrm{Cl} 1.14-21.89)^{34}$ to 5.9 (95\% Cl 1.6-21.3). ${ }^{36}$ Therefore, the current study adds on the prevalence of ED specifically for DFU population locally which was not done previously.

A study in Egypt found that psychological factors associated with FSD include fear, depression, fatigue, stress, anxiety and belief. ${ }^{18} \mathrm{~A}$ study by Kalmbach DA et al 2012 found that in cases involving healthy young adults showed that most sexual problems among women was more uniquely related to anhedonic depression; while for men, there are more mixed relationship of anxiety and depression. ${ }^{37}$ This study found that depressive symptoms and male gender were more likely to have SD in DM population. The result was affected by the main fact that participants who completed the questionnaire on SD were male who answered the IIEF 5 questionnaire.

In intimate relationships, both depression and anxiety are important psychological factors as causes and consequences of relationship distress. Previous local study found depressive symptoms to be positively associated with ED in aging population $^{16,17}$ and not for DM population, while a systematic review on risk factors for ED in Type 2 DM found that age, duration of illness, presence of peripheral neuropathy and $\mathrm{BMI}$ had positive association with erectile dysfunction. ${ }^{25}$

This study has several limitations. Firstly, the patients were recruited through a single centre i.e. the outpatient clinic of Medical and Orthopaedic departments of Hospital Raja Permaisuri Bainun Ipoh, Perak and hence the results cannot be generalized to other Malaysian population and also due to the modest sample size of patients. Secondly, this study did not distinguish between different types of diabetics, and most of the results involved Type 2 diabetics.

\section{CONCLUSION}

This study identified that the prevalence of depression and anxiety for diabetic patients, both with and without foot ulcer was similar. Male and lower education status was significantly associated with DFU. While depression, unemployment and smoking cigarette became significant risk factors for anxiety in DM, anxiety became significant risk factor for depression in DM. There was a significantly high prevalence of sexual dysfunction in DM with foot ulcer as both were the result of diabetes complication. For sexual dysfunction in DM, depression and male gender were significant risk factors. Overall, by identifying the prevalence and the risk factors involved, early detection and treatment for depression and anxiety may improve health outcomes, including sexual functions. However further studies are required to ascertain the directional nature of this relationship.

\section{Conflict of Interest \\ Nil}

\section{Abbreviation and symbols}

Body Mass Index, BMI

Confidence Interval $(\mathrm{Cl})$

Diabetic foot ulcer (DFU)

Diabetes Mellitus (DM)

Erectile Function (EF)

Erectile Dysfunction (ED)

Female Sexual Dysfunction (FSD)

Hospital Anxiety and Depression Scale (HADS)

Hospital Raja Permaisuri Bainun (HRPB)

Hypertension (HPT)

International Index for Erectile Function 5 (IIEF-5)

International Business Machine (IBM)

Interquartile Range (IQR)

Ischemic Heart Disease, IHD

Median (Med)

Medical Research and Ethics Committee (MREC)

Ministry of Health Malaysia $(\mathrm{MOH})$

National Medical Research Registry (NMRR)

Odd Ratio (OR)

Sexual Dysfunction (SD)

Statistical Package for Social Science (SPSS)

\section{REFERENCES}

1. Institute for Public Health (IPH): National health and morbidity survey 2015 (NHMS 2015).volume II: Non communicable diseases. Kuala Lumpur: Institute for Public Health (IPH); 2015.

2. Clinical Practice Guideline Management of Type 2 Diabetes Mellitus, $5^{\text {th }}$ edition. Ministry of Health Malaysia. 2015.

3. Farzamfar B, Nazari R and Bayanolhagh S. Diabetic Foot Ulcer. In Dr. Alexander Vitin, ed. Gangrene Management - New Advancements and Current Trends; InTech, 2013. Available at: http://www.intechopen.com/books/gangrenemanagement-new-advancements-and-currenttrends. Accessed July 25, 2016

4. Mezuk, B., Eaton, W. W., Albrecht, S., \& Golden, S. H. Depression and type 2 diabetes over the lifespan a meta-analysis. Diabetes care 2008; 31: 2383-2390.

5. Barnard, K. D., Skinner, T. C., \& Peveler, R. The prevalence of co-morbid depression in adults with Type 1 diabetes: systematic literature review. Diabetic Medicine 2006; 23: 445-448.

6. Atiq ur Rehman and Kazmi SF. Prevalence and Level of Depression, Anxiety and Stress among Patients with Type-2 Diabetes Mellitus. Ann. Pak. Inst. Med. Sci. 2015; 11: 81-86

7. Kaur, G., Tee, G. H., Ariaratnam, S., Krishnapillai, A. S., \& China, K. Depression, anxiety and stress symptoms among diabetics in Malaysia: a cross sectional study in an urban primary care setting. BMC family practice. 2013; 14: 69. 
8. Ganasegeran K, Renganathan P, Manaf RA, et al. Factors associated with anxiety and depression among type 2 diabetes outpatients in Malaysia: a descriptive cross-sectional single-centre study. BMJ Open 2014;4:e004794.doi:10.1136/ bmjopen-2014-004794

9. Ismail, K., Winkley, K., Stahl, D., Chalder, T., \& Edmonds, M. A Cohort Study of People with Diabetes and Their First Foot Ulcer The role of depression on mortality. Diabetes care, 2007; 30: 1473-1479.

10. Pearson, S and Nash, T and Ireland, V. Depression prevalence in people with diabetes and foot ulcers attending podiatry outpatient clinics, World Diabetes Congress 2013, Melbourne, Australia [Conference Extract]

11. Khuwaja AK, Lalani S, Dhanani R, et al. Anxiety and depression among outpatients with type 2 diabetes: a multi-centre study of prevalence and associated factors. Diabetol Metab Syndr 2010; 2:72.

12. Udovichenko Oleg V, Maximova Nadezhda V, Amosova Maria V, Yunilainen Olga A, Berseneva Eugenia A, Starostina Elena G. Prevalence and prognostic value of depression and anxiety in patients with diabetic foot ulcers and possibilities of their treatment Curr Diabetes Rev 2016. Available at www.Epubmed.com. Accessed May 5, 2016.

13. Pozzo MJ, Mociulsky J, Martinez ET, et al. Diabetes and Quality of Life: Initial Approach to Depression, Physical Activity, and Sexual Dysfunction. American Journal of Therapeutics 2016; 23:159-71.

14. Kamaralzaman S, Sidi H, Yau M, Budin SB, Sani A, Mohamed J. Sexual Function of Malay Women with Type 2 Diabetes Mellitus: A Preliminary Study. Asean Journal Of Psychiatry 2010; 11 :

15. Copeland, K. L., Brown, J. S., Creasman, J. M., et al. Diabetes Mellitus and Sexual Function in Middle-Aged and Older Women. Obstetrics and Gynecology, 2012; 120:331-340.

16. Khoo EM, Tan HM, and Low WY. Erectile dysfunction and comorbidities in aging men: An urban cross-sectional study in Malaysia. J Sex Med 2008;5:2925-2934

17. Tan, H. M., Tong, S. F., \& Ho, C. C. Men's health: sexual dysfunction, physical, and psychological health-is there a link?. The journal of sexual medicine 2012; 9: 663-671.

18. Ibrahim, Z. M., Ahmed, M. R., \& Ahmed, W. A. $S$. Prevalence and risk factors for female sexual dysfunction among Egyptian women. Archives of Gynecology and Obstetrics, 287(6), 1173-1180. doi: $10.1007 / \mathrm{s} 00404-012-2677-8$

19. Wagner WF. The dysvascular foot: a system of diagnosis and treatment. J Foot Ankle 1981;2:62 $-1221$.

20. Yahya F, Othman Z. Validation of the Malay Version of Hospital Anxiety and Depression Scale (HADS) in Hospital Universiti Sains Malaysia. International Medical Journal 2015; 22: 80-82.

21. Chapman Z, Shuttleworth CMJ, Huber JW. High levels of anxiety and depression in diabetic patients with Charcot foot. Journal of Foot and Ankle Research. 2014;7(1):1-8.

22. Fatt, Q. K., Atiya, A. S., Heng, N. G. C., \& Beng, C. C. Validation of the hospital anxiety and depression scale and the psychological disorder among premature ejaculation subjects. International journal of impotence research, 2007;19(3):321-325.

23. Lim, T. O., Das, A., Rampal, S., Zaki, M., Sahabudin, R. M., Rohan, M. J., et al. Crosscultural adaptation and validation of the English version of the International Index of Erectile Function (IIEF) for use in Malaysia. International Journal of Impotence Research, 2003; 15:329336.

24. Sidi, H., Abdullah, N., Puteh, S. E. W., \& Midin, $M$. The female sexual function index (FSFI): Validation of the Malay version. The journal of sexual medicine 2007; 4:1642-1654.

25. Binmoammar TA, Hassounah S, Alsaad S, Rawaf $S$, Majeed A. The impact of poor glycaemic control on the prevalence of erectile dysfunction in men with type 2 diabetes mellitus: a systematic review. JRSM open. 2016;7(3):2054270415622602.

26. Gardner SE, Hillis SL, Heilmann K, Segre JA, Grice EA. The neuropathic diabetic foot ulcer microbiome is associated with clinical factors. Diabetes. 2013;62(3):923-30.

27. Al Kafrawy N, Mustafa EA, Dawood AE-D, Ebaid $O$, Ahmed Zidane 0 . Study of risk factors of diabetic foot ulcers. Menoufia Medical Journal. 2014;27(1):28-34.

28. Collins MM, Corcoran P, Perry IJ. Anxiety and depression symptoms in patients with diabetes. Diabet Med 2009; 26:153-61.

29. Mazlina M, Shamsul AS, Saini Jeffery FA. Health Related Quality of Life in patient with Diabetic Foot Problems in Malaysia. Med J Malaysia 2011; 66:

30. Pedras, S, Carvalho, R, Pereira, MG. Sociodemographic and clinical characteristics of patients with diabetic foot ulcer. Revista da Associação Médica Brasileira 2016; 62: 171-178.

31. Iversen MM, Midthjell K, Tell GS, Moum T, Østbye T, Nortvedt MW, et al. The association between history of diabetic foot ulcer, perceived health and psychological distress: the Nord-Trøndelag Health Study. BMC Endocrine Disorders. 2009;9(1):18.

32. Cortelazzi D, Marconi A, Guazzi M, Cristina M, Zecchini B, Veronelli A, Cattalini C, Innocenti A, Bosco G, Pontiroli AE. Sexual dysfunction in premenopausal diabetic women: clinical, metabolic, psychological, cardiovascular, and neurophysiologic correlates. Acta Diabetol 2011; 50:911-917.

33. Gillani SW, Syed Sulaiman SA, Khalid Y, Sari YO, Baig $M$ and Amin A. Journal of Public Health and Epidemiology 2011; 3: 221-233.

34. Khoa Tuan V, Hoi Quoc Huynh, Nam Quang Tran, Aya Goto, Yuriko Suzuki, Khue Thy Nguyen. Erectile Dysfunction in Vietnamese Men with Type 2 DM. Journal of the ASEAN 
federation of endocrine societies 2014; 29.

Available at: www.asean-endocrinejournal.org. Accessed July 23, 2016.

35. Malavige LS, Levy JC. Erectile dysfunction in diabetes mellitus. J Sex Med. 2009; 6:1232-47.

36. Mutagaywa RK \& Lutale J, Aboud M, Benjamin Anathory Kamala. Prevalence of erectile dysfunction and associated factors among diabetic men attending diabetic clinic at Muhimbili National Hospital in Dar-es-Salaam, Tanzania Pan African Medical Journal. 2014;17:227 doi:10.11604/ pamj.2014.17.227.2695

37. Kalmbach, D. A., Ciesla, J. A., Janata, J. W. and Kingsberg, S. A. Specificity of Anhedonic Depression and Anxious Arousal with Sexual Problems Among Sexually Healthy Young Adults. Journal of Sexual Medicine 2012; 9: 505-513. 
